\title{
Down-under undergraduate optics and photonics laboratory
}

\section{Barry Perczuk, Michael Gal}

Barry Perczuk, Michael Gal, "Down-under undergraduate optics and photonics laboratory," Proc. SPIE 4588, Seventh International Conference on Education and Training in Optics and Photonics, (28 May 2002); doi: 10.1117/12.468716

SPIE Event: Education and Training in Optics and Photonics 2001, 2001, Singapore, Singapore 


\title{
A down-under undergraduate optics and photonics laboratory
}

\author{
Barry Perczuk and Michael Gal \\ School of Physics, The University of New South Wales, Sydney, NSW 2052, Australia
}

\begin{abstract}
Our senior undergraduate laboratory offers 14 experiments in optics and photonics, including experiments on acoustooptics, properties of lasers, holography, optical fibre sensors and communications. Six of the experiments, and a mandatory assignment on laser safety, are individually completed by each student in a one semester course. A brief description of the experimental course and of each experiment is given, together with more detailed descriptions of the 'Fourier optics' and "Photoluminescence of semiconductor quantum wells' experiments.
\end{abstract}

Keywords: optics/ photonics laboratory, Fourier optics, photoluminescence, quantum well

\section{HISTORICAL INTRODUCTION}

In the late 1970s several traditional optics experiments were offered as part of third year (i.e. last undergraduate year) experimental physics courses at the University of New South Wales (UNSW). These were experiments on the properties of HeNe laser light, polarisation of light, Fourier optics and Fourier transform spectroscopy.

In the mid 1980s interest in optics increased in the school and several new experiments were added, namely experiments on optical fibres, holography, optical spectroscopy, photodetector and light source characteristics and acousto-optics. There were now sufficient experiments to offer a separate, semester long experimental course purely in lasers and optics.

As interest in optical fibre communications increased, we obtained grants in the early 1990s to set up a separate Laser and Optoelectronics laboratory to teach the more modern aspects of optoelectronics. Experiments on optical fibre communications, optical fibre sensors, optical time domain reflectometry (OTDR) the Neodymium YAG laser and photoluminescent spectroscopy of semiconductor quantum wells, were added at this stage.

Since the beginning of the 1990s a great deal of undergraduate interest has been shown in this experimental course. It has been successfully completed by many students in disciplines ranging from medical technology to electrical and mechanical engineering.

\section{COURSE STRUCTURE}

Safety in the laser laboratory is emphasized from the very beginning of the course. During their first class students attend a short talk and demonstration on laser safety issues and are then required to complete a laser safety assignment, with problems and questions based on the Australian Standard for Laser Safety. Safe practice is then constantly checked by the demonstrators throughout the duration of the course.

During the teaching semester of 14 weeks, students attend one 4 hour laboratory session weekly and are expected to complete 5 or 6 experiments of their choice out of the 14 offered. Experiments vary in duration from 6 to 12 hours of laboratory work, with students expected to devote as much time again for each experiment outside the laboratory, reading references, analysing data and presenting their results and conclusions. There is no special order in which experiments are carried out, although some of the more basic are prerequisites for a few of the more complex ones (e.g. the 'Optical fibres' experiment needs to be completed before attempting 'Optical fibre communications' or 'OTDR'). Students work individually, or in pairs if they prefer. The laboratory operates, as much as practicable, as an 'open' laboratory where students can work at their own pace and are able to come in at various times, outside of their scheduled laboratory sessions, to work on experiments. 
There is no textbook assigned for the course. Students are issued a set of notes for each experiment, which includes fairly explicit experimental instructions, questions and a list of relevant references. Additionally, information folders, which may be borrowed for each of the experiments, contain necessary equipment specifications, operating instructions and other useful data.

Students keep a record of their experimental work in laboratory notebooks which are expected to be 'work books' containing all raw results, comments on experimental conditions and calculations. At the completion of each experiment students are required to analyse and present their results independently. Assessment is based on an interview/ feedback session with a senior academic demonstrator, who, among other things, examines each student's laboratory notebook and their answers to the set question. Students often comment that they learn as much during the feedback interviews as in the actual laboratory sessions. No formal reports are required for the course.

\section{SUMMARY OF THE EXPERIMENTS}

The experiments offered can be divided into four topic groups, viz. lasers and light; diffraction and polarisation of light; spectroscopy; fibre optics.

A summary of each experiment follows:

\section{Properties of HeNe lasers and their light}

Some properties of HeNe lasers and their light are studied including: wavelengths emitted, spatial and temporal coherence, longitudinal modes, output power stability, polarisation, speckle phenomena.

\section{The Neodymium YAG laser}

A Neodymium YAG laser, pumped by a semiconductor laser diode, is set up on an optical table, using discrete optical components. The properties of the light emitted by both the laser driver diode and the YAG laser are studied using a computer based fibre optic spectrometer.

\section{Holography}

Introduction to the principles and techniques of holography. Transmission and white-light reflection holograms of suitable objects, as well as a simple holographic transmission interferogram, are produced.

\section{Fourier optics}

An introduction to optical image processing, employing a CCD camera and a frame grabber to capture images: lenses as optical Fourier transform computers, spatial frequencies and resolution, effect of filters in the diffraction plane on the final image, manufacture of simple blocking filters, frequency doubling and tripling.

\section{Acousto-optics}

Light diffraction by acoustic waves in transparent media. Two commercial acousto-optic modulators /deflectors (flint glass, lead molybdate) are used to gain an insight into the similarities and differences between Bragg and Raman-Nath diffraction.

\section{Polarisation of light}

A photodiode detector is used to quantitatively study linearly, circularly and elliptically polarised HeNe laser light produced by: scattering in suspensions, linear polarisers, quarter wave plates, circular polarisers, reflection from dielectrics (Fresnel's laws) and reflection from metals.

\section{Optical spectroscopy}

The aim is to understand the optical system and operation of a commercial UV-visible spectrometer, which is then used to measure the light transmission characteristics of thin films, optical filters, sunglasses and various sun screen lotions. 


\section{Photo-detector and light source characteristics}

A computer driven $0.22 \mathrm{~m}$ spectrometer is used to study the spectra of various different light sources (projector lamp, fluorescent lamp, Hg lamp, LEDs, HeNe laser, IR laser diode). The spectral output of the projector lamp is measured with a thermopile detector and the relative spectral responses of several photo-detectors are then measured and compared.

\section{Photoluminescence spectroscopy of semiconductor quantum wells}

A computer controlled $0.32 \mathrm{~m}$ monochromator is used to measure the photoluminescent spectrum excited by $633 \mathrm{~nm}$ HeNe light in two samples of GaAs at 77K; one a bulk single crystal, the other a $10 \mathrm{~nm}$ layer sandwiched between thick GaAlAs layers. The measured difference in energy band gaps due to the quantum size effect, quantifies the discussion of semiconductor quantum wells.

\section{Fourier transform spectrometry}

The Michelson interferometer as a spectrometer; Fourier transform pair relationship between the interferogram and the incident spectral distribution. Spectra of various light sources, effect of colour filters and detectors with different spectral responses. Limitations: resolution, aliasing, noise.

\section{Optical fibres}

The fundamental properties of multimode and single mode fibres are studied. Cleaving of fibre ends, coupling of laser light into fibres, measurement of numerical aperture and attenuation coefficient of a multimode fibre, investigation of the farfield power distribution of a single mode fibre.

\section{Optical fibre sensors}

Several intensity sensors (proximity, pressure, liquid level), which exploit the optical properties of multimode fibres, and a single mode interferometric phase sensor are constructed. The sensitivity and resolution of these devices is studied.

\section{Optical fibre communication}

The basic optical characteristics of two semiconductor light sources (a light emitting diode and an injection laser diode) are first studied, A fibre optic communication link, with these two light sources as the analog inputs, is then constructed, using thin film filters to demonstrate wavelength division multiplexing and demultiplexing.

\section{Optical time domain reflectometry}

An OTDR is set up on the optical table, using discrete optical components. It is then used to measure attenuation and splicing losses in optical fibre spools.

A somewhat more detailed description of two of the most popular experiments now follows.

\section{QUANTUM WELLS EXPERIMENT}

This experiment presents students with a very useful combination - a modern spectrometry system applied to the study of the properties of a quantum well semiconductor.

A schematic diagram of the apparatus used in the measurement of the photoluminescent spectrum excited from semiconductor samples is shown in Figure 1. To align the light gathering optics and the spectrometer, a red LED is initially located at the sample position and its emission spectrum is measured, using the spectrometer and a DC method to record the silicon detector output. The output light intensity of the LED is then reduced, to show the limitations of the DC method. The chopper and lock-in amplifier are introduced at this stage to give the student an appreciation of how small, periodic signals buried in noise, can be retrieved. 


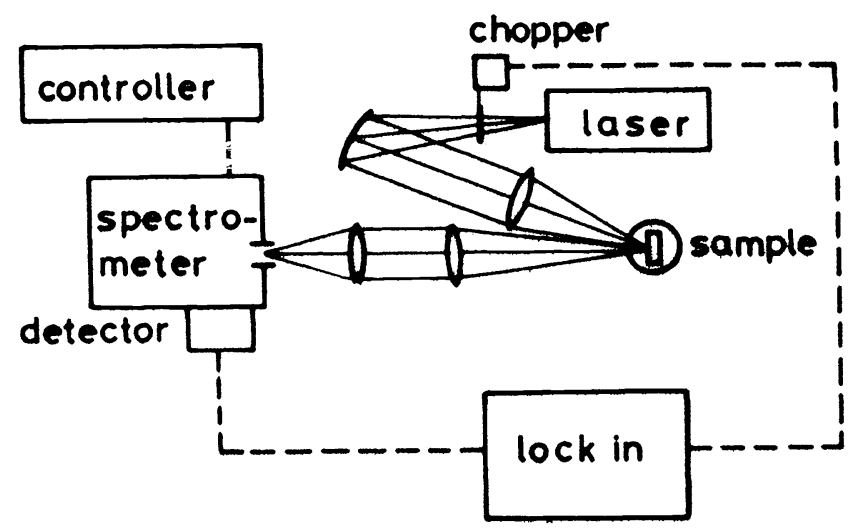

Fig. 1: Schematic of the photoluminescence measurement apparatus

The red LED is now replaced by the sample cryostat and the light beam (chopped) from a HeNe laser is focussed onto the semiconductor sample, which is cooled to $77 \mathrm{~K}$ to increase the luminescent yield. The luminescence excited from the sample is then focussed onto the entrance slit of the spectrometer, whose output is measured using the detector/ lock-in combination. The luminescence from a GaAs /AlGaAs quantum well sample is measured first. For purposes of comparison, the luminescence of a bulk GaAs sample is also measured using the same set up. From the measured difference in the luminescent wavelengths (i.e. band gaps) of the two samples, the effective mass of the conduction electrons in the quantum well sample can then be calculated.

Useful learning outcomes of this experiment, apart from reinforcing the quantum mechanical principles encountered in lectures, include experience with: a modern optical system employing folded beam geometry and beam steering; a chopped light source and lock-in detection; a computer controlled spectrometer; a liquid nitrogen cryostat with a vacuum pumping system.

\section{FOURIER OPTICS EXPERIMENT}

The purpose of this experiment is to allow students to actually 'see' various properties of Fourier transforms. It is the favourite experiment with many students.

A $1 \mathrm{~mm}$ diameter HeNe laser beam is filtered, expanded and collimated to give a $50 \mathrm{~mm}$ diameter beam parallel to a 3 metre long optical rail. Suitably sized transparencies of various objects (e.g. a grid, a half-tone photograph, cloud chamber tracks) are inserted into this beam and the diffraction patterns, formed in the focal plane of an accurately ground $\mathrm{f} / 5$ plano-convex lens (the 'transform' lens), positioned on the optical rail, one focal length away from the object transparency, may be studied or filtered. Slits, lines, apertures and dots of various sizes, suitably mounted on slide frames, are available to carry out both pass and rejection filtering operations. After filtering, the final image can be reconstructed using a matching plano-convex lens, mounted on the same optical rail two focal lengths away from the 'transform' lens . A CCD camera, coupled to a frame grabber, is used to capture the light intensity distribution either in the diffraction plane or in the final image plane. Image processing software, including the facility to perform two dimensional fast Fourier transforms (FFTs), can then be used to simulate various filtering operations or to analyse the final image.

In this laboratory exercise, apart from experimentally confirming most of the main properties of two dimensional Fourier transforms covered in lecture courses (e.g. the shift theorem, Babinet's theorem, the Fourier inversion theorem), students are able to immediately see the effects of low and high pass and rejection filtering, directional pass and rejection filtering 
and are able to carry out frequency doubling and tripling by appropriately manufacturing their own spatial filters. Judging from student comments, this experiment gives students a real 'feel' for Fourier transforms, which escapes many of them in lectures on the topic.

Useful learning outcomes also include experience with: a coherent optical imaging lens system; spatial filtering; a CCD camera with frame grabber and image processing software.

\section{CONCLUSIONS}

The experimental optics/photonics courses offered at UNSW have rated consistently highly on student surveys. Offered as electives, they have attracted as many students as the compulsory general physics laboratory courses. Two, one semester experimental courses, incorporating a selection of the above experiments, are now required as part of a Masters of Science and Technology in Photonics and Electronics degree by course work, introduced at the beginning of 2001. There is a lot of interest in this program from graduates working in other scientific/engineering disciplines but wishing to retrain for employment in the photonics industry.

In the near future we are intending to extend the range of available experiments by introducing experiments on:

laser diode characteristics; Erbium doped fibre amplifiers; dense wave division multiplexing using Bragg fibre gratings.

\section{ACKNOWLEDGEMENT}

Financial support from the New South Wales Education and Training Foundation, for the setting up of the teaching laboratory, is gratefully acknowledged. 